\title{
Linear Array HPV Genotyping Test
}

National Cancer Institute

\section{Source}

National Cancer Institute. Linear Array HPV Genotyping Test. NCI Thesaurus. Code C120297.

A proprietary PCR technique that uses a pool of primers to amplify a 450 bp region in the L1 region of the HPV genome. It is capable of detecting 37 high- and low-risk human papillomavirus genotypes. 